\title{
Atrioventricular valve repair in patients with functional single-ventricle physiology: Impact of ventricular and valve function and morphology on survival and reintervention
}

\author{
Osami Honjo, MD, PhD, Cori R. Atlin, BA, Luc Mertens, MD, PhD, Osman O. Al-Radi, MD, MSc, \\ Andrew N. Redington, MD, Christopher A. Caldarone, MD, and Glen S. Van Arsdell, MD
}

\begin{abstract}
Objective: This study was to determine whether atrioventricular valve repair modifies natural history of single-ventricle patients with atrioventricular valve insufficiency and to identify factors predicting survival and reintervention.
\end{abstract}

\begin{abstract}
Methods: Fifty-seven (13.5\%) of 422 single-ventricle patients underwent atrioventricular valve repair. Valve morphology, regurgitation mechanism, and ventricular morphology and function were analyzed for effect on survival, transplant, and reintervention with multivariate logistic and Cox regression models. Comparative analysis used case-matched controls.

Results: Atrioventricular valve was tricuspid in $67 \%$ and common in $28 \%$. Ventricular morphology was right in $83 \%$. Regurgitation mechanisms were prolapse $(\mathrm{n}=24,46 \%)$, dysplasia $(\mathrm{n}=18,35 \%)$, annular dilatation $(\mathrm{n}=8,15 \%)$, and restriction or cleft $(\mathrm{n}=2,4 \%)$. Postrepair insufficiency was none or trivial in $14(26 \%)$, mild in $33(61 \%)$, and moderate in $7(13 \%)$. Survival in repair group was lower than in matched controls (78.9\% vs $92.7 \%$ at 1 year, $68.7 \%$ vs $90.6 \%$ at 3 years, $P=.015)$. Patients with successful repair and normal ventricular function had equivalent survival to matched controls $(P=.36)$. Independent predictors for death or transplant included increased indexed annular size $(P=.05)$, increased cardiopulmonary bypass time $(P=.04)$, and decreased postrepair ventricular function $(P=.01)$. Ventricular dilation was a time-related factor for all events, including failed repair.
\end{abstract}

Conclusions: Survival was lower in single-ventricle patients operated on for atrioventricular valve insufficiency than in case-matched controls. Patients with little postoperative residual regurgitation and preserved ventricular function had equivalent survival to controls. Lower grade ventricular function and ventricular dilation correlated with death and repair failure, suggesting that timing of intervention may affect outcome. (J Thorac Cardiovasc Surg 2011;142:326-35)

丹 Supplemental material is available online.

Significant atrioventricular valve (AV) insufficiency in single-ventricle physiology was one of the contraindications for staged single-ventricle palliation in the past. ${ }^{1}$ Introduction of various AV repair techniques ${ }^{2,3}$ and application of AV repair during the staged palliation made Fontan completion among those patients feasible. Nevertheless, despite continued improvement in outcomes for patients at

From The Labatt Family Heart Centre, The Hospital for Sick Children, and The University of Toronto, Toronto, Ontario, Canada.

Disclosures: Authors have nothing to disclose with regard to commercial support.

Read at the 90th Annual Meeting of The American Association for Thoracic Surgery, Toronto, Ontario, Canada, May 1-5, 2010.

Received for publication May 4, 2010; revisions received Sept 5, 2010; accepted for publication Nov 2, 2010; available ahead of print May 18, 2011

Address for reprints: Glen S. Van Arsdell, MD, Division of Cardiovascular Surgery, The Hospital for Sick Children, 555 University Ave, Toronto, Ontario, Canada, M5G 1X8 (E-mail: glen.vanarsdell@sickkids.ca).

$0022-5223 / \$ 36.00$

Copyright (c) 2011 by The American Association for Thoracic Surgery

doi:10.1016/j.jtcvs.2010.11.060 higher risk, ${ }^{4,5} \mathrm{AV}$ insufficiency is still associated with failure of the cavopulmonary circulation. ${ }^{2,6,7}$

Mechanisms of AV insufficiency in a single-ventricle physiology are complex and multifactorial. ${ }^{8,9}$ Chronic volume overload with a physiology dependent on systemicto-pulmonary shunt causes progressive ventricular dilatation. Subsequent annular dilatation may cause central malcoaptation, leading to AV insufficiency. AV leaflets and the subvalvular apparatus in functionally univentricular hearts are often abnormal. ${ }^{10}$ A tricuspid valve as the systemic AV appears to be more prone to functional tricuspid valve insufficiency than does a mitral valve. Reduced ventricular function is also associated with the development of AV insufficiency.

We hypothesized that ventricular function, AV morphology, and mechanism of insufficiency all play important roles in determining patient outcome. In this study we attempted to answer 2 questions: (1) What is the relationship between ventricular function and success or failure of AV repair? (2) Does AV repair modify the natural history of the single-ventricle patients with significant AV insufficiency? Cohort and case-control analyses were performed. 


\author{
Abbreviations and Acronyms \\ $\mathrm{AV}=$ atrioventricular valve \\ BCPS $=$ bidirectional cavopulmonary shunt \\ BSA $=$ body surface area \\ $\mathrm{CPB}=$ cardiopulmonary bypass \\ TEE $=$ transesophageal echocardiography
}

\section{MATERIALS AND METHODS}

We reviewed the cases of all patients who underwent staged singleventricle palliation between January 1998 and December 2008 at The Hospital for Sick Children, Toronto, Ontario, Canada. The institution's research ethics board approved the study and waived the requirement for patient consent. Of the 442 patients who underwent single-ventricle palliation, $57(13.5 \%)$ also underwent AV repair. Diagnoses and preoperative characteristics are shown in Table E1. AV morphologies, types of $\mathrm{AV}$ abnormalities, and ventricular function grades are shown in Table 1.

\section{Threshold and Timing of AV Repair}

Moderate or greater AV insufficiency was our clear indication for adding AV repair to an already planned staged operation. We inspected and attempted to repair AVs with mild to moderate insufficiency because we believe that those AVs are often structurally abnormal. Details of the timing of repair in this case series are outlined in Table E1. We were hesitant to perform a complex AV repair at the stage III procedure and instead tended to perform a separate procedure for AV repair in cases of severe interstage II and III AV regurgitation. Our preferred time for repair was at the time of the stage II bidirectional cavopulmonary shunt (BCPS). Valve repair in a neonate was rare in this case series. Some neonates with single-ventricle physiology and severe AV insufficiency were instead assigned to undergo primary heart transplant.

\section{Surgical Techniques}

Standard cardiopulmonary bypass (CPB) was established, with mild to moderate hypothermia. Deep hypothermic circulatory arrest was used only when performing other concomitant procedures, such as aortic arch reconstruction and pulmonary vein repair. An initial saline injection test determined the mechanism of AV insufficiency. Special attention was paid to the annular dimension, commissural leak, prolapse or restriction of the leaflets, and leaflet and subvalvular abnormalities. Local annuloplasty and commissuroplasty were the 2 main techniques used for annular dilatation, leaflet prolapse, or both. We typically used 5-0 polypropylene horizontal mattress sutures to reduce the annular size along the commissures where the regurgitant jet mainly arose. The maneuver could serve as a functional commissuroplasty on the corresponding commissure. Anatomic commissuroplasty was achieved with 6-0 or 7-0 polypropylene simple sutures placed on the leaflet tissue as an edge-to-edge approximation, such as one would use to close a cleft. We occasionally obliterated the posterior leaflet of the tricuspid valve, as described by Bove and colleagues. ${ }^{8,11}$ An artificial annuloplasty ring was only used in patients with an adult-sized annulus. Neither a semicircular nor a circular annuloplasty was used in our series. A localized or generalized prolapse with essentially normal chordae and subvalvular apparatus on the adjacent leaflet was treated with an edge-to-edge commissure approximation ${ }^{12}$ to attach the prolapsed region to the nonprolapsed region. A local annuloplasty was usually added. Clefts were primarily closed with interrupted polypropylene sutures. Nodular dysplastic leaflet edges were made as smooth as possible by the attachment of the nodules to each other. Dysmorphic leaflets with serpiginous edges were treated in a similar manner to improve the linearity of the coaptation area. The definition of AV dysplasia used was myxoid thickening, nodular irregularity of the leaflet edge, or both, whereas dys- morphic leaflet was considered to be the presence of 1 or more relatively large gaps or accessory commissures of the leaflet, resulting in malcoaptation or localized prolapse. Intraoperative transesophageal echocardiography (TEE) was routinely performed, and any less than moderate residual AV insufficiency found on TEE was acceptable. Our intent was to revise the repair in cases of moderate or greater residual regurgitation. Replacement of an $\mathrm{AV}$ was performed in cases of greater than moderate residual regurgitation or if surgical inspection showed irreparable leaflet abnormalities. A standard bileaflet mechanical valve was used.

\section{Echocardiographic Evaluation}

Original echocardiographic images were evaluated by an echocardiographic investigator (L.M.) who was blinded to the original echocardiographic report and to clinical outcome. Morphologies of the AVs and ventricles were determined. Structural abnormalities of the AVs, including annular dilatation, cleft, prolapse, chordal elongation or deficiency, restriction, dysplasia, and papillary muscle abnormalities, were assessed (Table 1). Both primary and secondary mechanisms of AV insufficiency were determined. The AV insufficiency, stenosis, and ventricular function grades we used are listed in Table E2. Ventricular dimension and AV annular dimension were measured from the apical 4-chamber view and were indexed by body surface area (BSA) as millimeters per square meter.

\section{Case-Control Study}

Three control patients were matched to each study patient on the basis of diagnosis, body weight, BSA, ventricular and AV morphologies, and type, number, and timing of the staged palliations. Body weight and BSA were matched at prerepair measurement. A single best fit was then chosen according to ventricular function and AV insufficiency grades and verified by actual echocardiographic imaging review. In terms of ventricular function, an equivalent grade or a single grade difference was accepted. Freedom from death or transplant and freedom from all events were compared between the matched control group and the valve repair group.

\section{Outcome Assessment}

Failed AV repair was defined as evidence of more than moderate residual AV insufficiency on intraoperative TEE. In-hospital death was defined as all deaths that occurred after the AV repair and within 30 days or the same hospitalization. Two composite outcomes-freedom form death or transplant and freedom from all events, including death, transplant, repeated repair, or replacement-were defined. These outcomes were compared with respect to the diagnosis, ventricular and AV morphologies, primary mechanism, and timing of surgery. The cohort was separated into 4 subgroups according to postrepair AV and ventricular functions (Table E2). The outcomes were then compared among these 4 groups. The predictors for repeated repair or replacement were analyzed among the patients who survived without transplant longer than 6 months after the initial AV repair.

\section{Statistical Analysis}

Data are presented as mean \pm SD. Differences between the study patients and the control patients were analyzed with a paired $t$ test or a Wilcoxon signed rank test. Frequencies of the events were compared between the groups with a $\chi^{2}$ test. Correlations between preoperative and postoperative values were analyzed with the Pearson correlation coefficient. Differences in ventricular function grades over time were analyzed with 1-way analysis of variance. Freedom from death or transplant and freedom from all events (death, transplant, repeated repair, or replacement) were analyzed with Kaplan-Meier survival analysis, with a log-rank test used for comparisons between the groups. Multivariate logistic regression of the entire cohort was used to determine independent variables of risk for the end points of freedom from death or transplant and freedom from all events (death, transplant, repeated repair, or replacement). Cox regression models were used to determine the timerelated predictors for the outcomes. 
TABLE 1. Echocardiographic findings: Atrioventricular valve morphology, insufficiency, and ventricular function

\begin{tabular}{|c|c|c|c|c|}
\hline Variables & $\mathbf{R V}$ & $\mathbf{L V}$ & Indeterminate & Total \\
\hline \multicolumn{5}{|l|}{ Valve morphology } \\
\hline Mitral valve & 0 & 2 & 0 & $2(4 \%)$ \\
\hline Tricuspid valve & 38 & 0 & 0 & $38(67 \%)$ \\
\hline Common AV & 9 & 6 & 1 & $16(28 \%)$ \\
\hline Indeterminate & 0 & 0 & 1 & $1(2 \%)$ \\
\hline \multicolumn{5}{|l|}{ AV insufficiency } \\
\hline Mild to moderate & 4 & 1 & 1 & $6(11 \%)$ \\
\hline Moderate & 31 & 5 & 1 & $37(65 \%)$ \\
\hline Severe & 12 & 2 & 0 & $14(25 \%)$ \\
\hline \multicolumn{5}{|l|}{ Valve abnormalities } \\
\hline Annular dilatation & 35 & 5 & 1 & $41(77 \%)$ \\
\hline Cleft & 7 & 3 & 1 & $11(21 \%)$ \\
\hline Prolapse & 24 & 2 & 1 & $27(51 \%)$ \\
\hline Chordal elongation & 21 & 1 & 0 & $22(42 \%)$ \\
\hline Restriction & 24 & 2 & 0 & $26(49 \%)$ \\
\hline Dysplasia & 21 & 5 & 1 & $27(52 \%)$ \\
\hline Papillary muscle abnormality & 4 & 0 & 0 & $4(8 \%)$ \\
\hline \multicolumn{5}{|l|}{ Primary mechanism } \\
\hline Annular dilatation & 8 & 0 & 0 & $8(15 \%)$ \\
\hline Prolapse & 22 & 2 & 0 & $24(46 \%)$ \\
\hline Restriction & 1 & 0 & 0 & $1(2 \%)$ \\
\hline Dysplasia & 12 & 5 & 1 & $18(35 \%)$ \\
\hline Cleft & 1 & 0 & 0 & $1(2 \%)$ \\
\hline \multicolumn{5}{|l|}{ Secondary mechanism } \\
\hline Annular dilatation & 11 & 2 & 0 & $13(25 \%)$ \\
\hline Prolapse & 3 & 0 & 1 & $4(8 \%)$ \\
\hline Restriction & 4 & 0 & 0 & $4(8 \%)$ \\
\hline Dysplasia & 5 & 0 & 0 & $5(10 \%)$ \\
\hline Cleft & 21 & 5 & 0 & $26(50 \%)$ \\
\hline \multicolumn{5}{|l|}{ Ventricular function } \\
\hline Normal & 27 & 6 & 1 & $34(64 \%)$ \\
\hline Mildly reduced & 14 & 1 & 0 & $15(28 \%)$ \\
\hline Moderately reduced & 3 & 0 & 0 & $3(6 \%)$ \\
\hline Severely reduced & 1 & 0 & 0 & $1(2 \%)$ \\
\hline \multicolumn{5}{|l|}{ Ventricular dilatation } \\
\hline Normal & 0 & 1 & 1 & $2(4 \%)$ \\
\hline Mildly dilated & 1 & 0 & 0 & $1(2 \%)$ \\
\hline Moderately dilated & 35 & 6 & 0 & $41(77 \%)$ \\
\hline Severely dilated & 9 & 0 & 0 & $9(11 \%)$ \\
\hline
\end{tabular}

Data represent numbers of patients. $R V$, Right ventricle; $L V$, left ventricle; $A V$, atrioventricular valve.

\section{RESULTS}

Hypoplastic left heart syndrome and unbalanced atrioventricular defect accounted for $59 \%$ and $19 \%$ of cases, respectively (Table E1). The right ventricle was the dominant ventricle in $83 \%$ of cases. More than half the patients $(59 \%)$ underwent AV repair at stage II palliation. Ten $(17 \%)$ patients underwent $\mathrm{AV}$ repair as a stand-alone procedure between stage II and stage III operations.

There were 3 patients with a single-ventricle physiology who were listed for primary transplant, primarily as a result of significant AV insufficiency in the study period.

\section{AV Morphology and Mechanism of Insufficiency}

The most common AV type was tricuspid valve (67\%), followed by common AV (28\%). Mitral valve morphology was seen in 2 patients $(4 \%)$. Preoperative AV insufficiency, ventricular function and dilatation, and types of $\mathrm{AV}$ abnormalities are shown in Table 1 . The most common primary mechanism was prolapse $(n=24,46 \%)$, followed by dysplasia $(\mathrm{n}=18,35 \%)$. Pure annular dilatation accounted for $15 \%(n=8)$ of cases. Twenty six patients $(57 \%)$ had 2 major mechanisms of $\mathrm{AV}$ insufficiency. A cleft was the most common secondary mechanism for AV insufficiency $(50 \%)$. Preoperative ventricular function was mildly reduced in 15 patients $(28 \%)$ and moderately or severely reduced in 4 patients $(8 \%)$.

\section{Immediate Outcomes of AV Repair}

More than 1 technique was used for most patients, with annuloplasty being the most common $(85 \%)$, followed by commissuroplasty $(54 \%)$ and cleft closure $(33 \%$; Table E3). Three (4\%) patients underwent artificial ring annuloplasty. Postrepair AV insufficiency was none or trivial in 14 patients $(26 \%)$, mild in 33 patients $(61 \%)$, and moderate in 7 patients $(13 \%)$. Valve insufficiency grade was significantly reduced with repair (prerepair $2.1 \pm 0.57$ vs postrepair $0.84 \pm 0.62, P=.0001$; Figure E1). Postrepair AV stenosis was mild in 9 patients $(15 \%)$ and moderate in 1 patient $(2 \%)$. Postrepair ventricular function was normal in 36 patients $(63 \%)$, mildly reduced in 10 patients $(17 \%)$, moderately reduced in 7 patients $(13 \%)$, and severely reduced in 4 patients $(7 \%)$.

\section{Independent Predictors of Poor Valve and Ventricular Function}

Preoperative ventricular dilatation was a predictor for a failed repair (more than moderate residual AV insufficiency, $P=.03$ ). The predictors for moderately or severely reduced ventricular function as seen on TEE included deep hypothermic circulatory arrest $(P=.03)$ and annular dilatation as the primary mechanism of insufficiency $(P=.04)$. Prerepair ventricular function was correlated with postrepair ventricular function $(r=.569, P=.01)$ but did not predict postrepair ventricular dysfunction (difference not significant). Preoperative ventricular dilatation was weakly positively correlated with postoperative ventricular function $(r=.3, P=.04)$. Six of 8 patients who had annular dilatation as the primary mechanism $(75 \%)$ had postrepair ventricular dysfunction.

\section{Survival}

There were 8 in-hospital deaths (14\%; Table E4). Two patients who had moderate residual AV insufficiency died, 1 of sudden cardiac arrest and the other of progressive ventricular failure. There were 3 early deaths of patients with 
Freedom from death/transplant by diagnosis

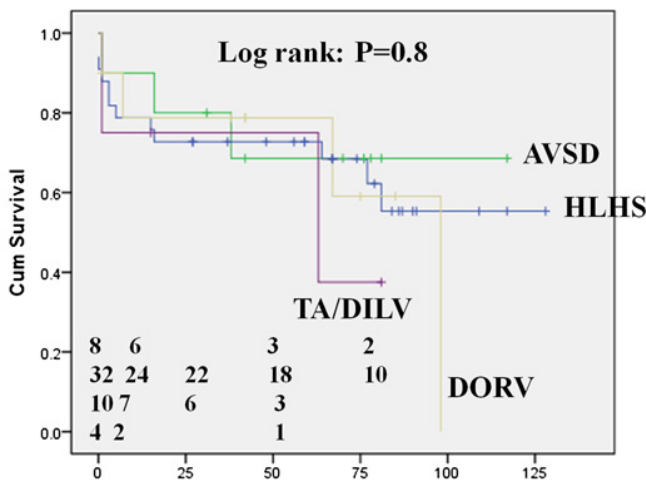

A

Months from valve repair

Freedom from death/transplant by AV valve morphology

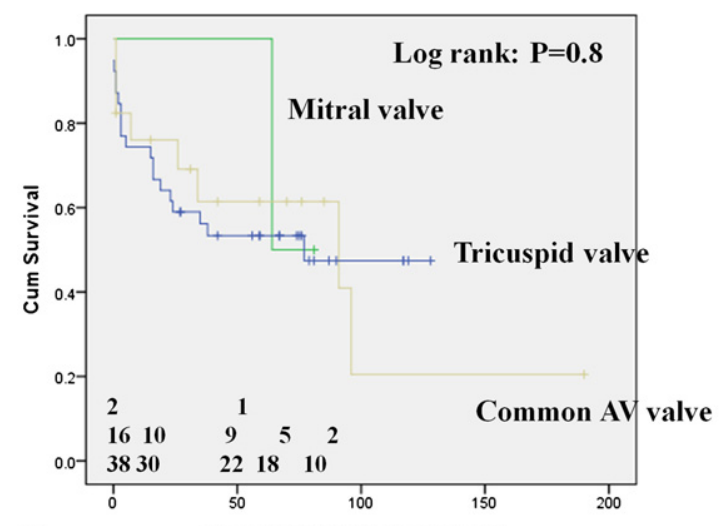

C

Months from valve repair

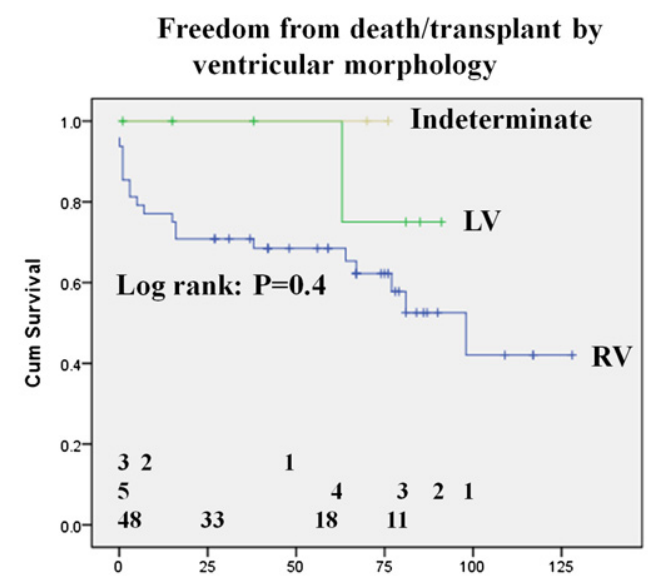

B Months from valve repair

Freedom from death/transplant by post-repair valve and ventricular function

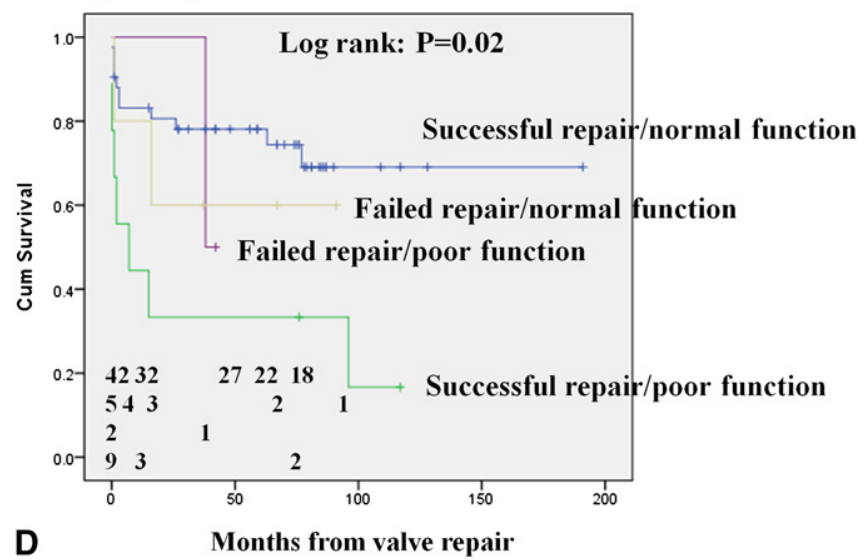

FIGURE 1. Kaplan-Meier survival analyses of the single-ventricle patients undergoing atrioventricular ( $A V)$ valve repair. Cumulative (Cum) survivals are given by diagnosis (A), by ventricular morphology (B), by atrioventricular valve morphology (C), and among 4 subgroups determined according to postrepair residual atrioventricular valve insufficiency and ventricular function (D). AVSD, Atrioventricular septal defect; $H L H S$, hypoplastic left heart syndrome; $T A / D I L V$, tricuspid atresia with double-inlet left ventricle; $D O R V$, double-outlet right ventricle; $L V$, left ventricle; $R V$, right ventricle.

hypoplastic left heart syndrome who had poor progress after stage I palliation and underwent early stage II palliation with AV repair. There were 9 late deaths (15\%), including 3 cardiac-related deaths.

No differences were found in survival among diagnoses $(P=.8)$, ventricular $(P=.4)$ or AV morphology $(P=.8)$, mechanism of insufficiency $(P=.16)$, or timing of surgery $(P=.4$; (Figure 1, $A, B$, and $C$ ). The survival according to postrepair ventricular function and $\mathrm{AV}$ insufficiency divided into groups showed the group who had successful repair with normal ventricular function to have significantly better survival than other groups $(P=.02$; Figure $1, D)$.

\section{Risk Factor Analysis}

Independent predictors for death or transplant included increased indexed AV annular size $(P=.05)$, increased CPB time $(P=.04)$, and decreased postrepair ventricular function $(P=.01)$. The predictors for all events were increased indexed $\mathrm{AV}$ annular $(P=.006)$ and ventricular $(P=.02)$ dimensions, increased aortic crossclamp $(P=.01)$ and CPB $(P=.002)$ times, and decreased postrepair ventricular function $(P=.02)$. Failed repair did not reach statistical significance $(P=.07)$ as a predictor. Repair era was not identified as a predictor for survival (difference not significant).

Cox regression showed that predictors for death or transplant included CPB time $(P=.02)$, annular dilatation as a primary mechanism $(P=.02)$, postrepair $\mathrm{AV}$ insufficiency grade $(P=.03)$, and postrepair ventricular function $(P=.02)$. Predictors for all events (death, transplant, and reoperation) included BSA $(P=.008)$, CPB time $(P=.001)$, and preoperative ventricular dilatation $(P=.05)$. None of the preoperative anatomic characteristics were shown to be predictors for death or transplant. 


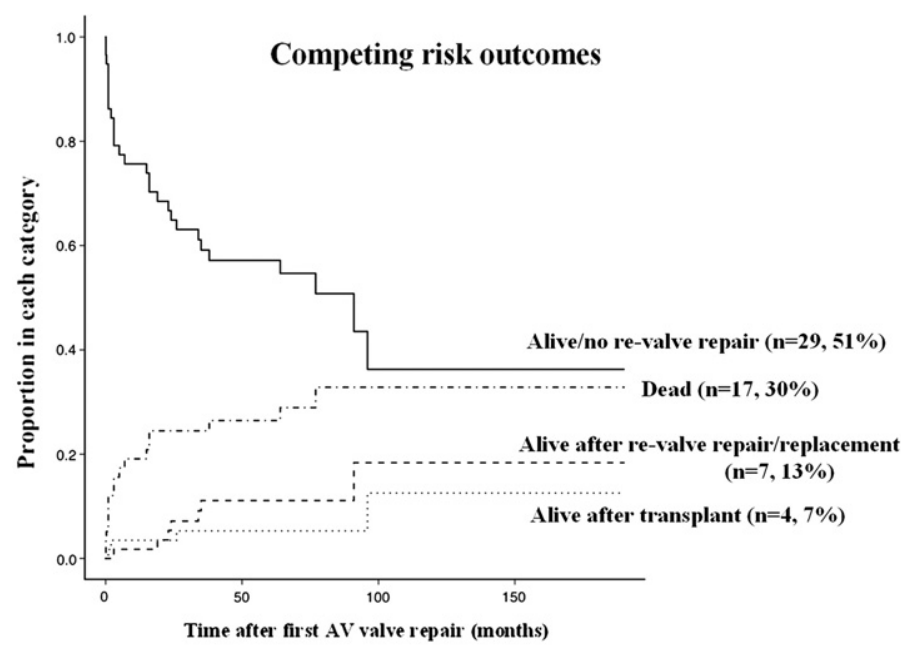

A

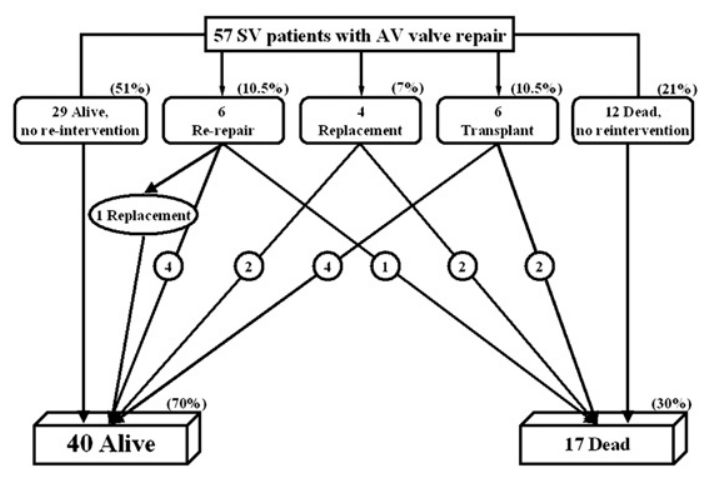

B

FIGURE 2. A, Competing risk outcomes for the entire cohort. B, The flow chart for the 57 patients who underwent atrioventricular $(A V)$ valve repair. $S V$, Single ventricle.

A competing risk outcome of the entire cohort is shown in Figure 2, $A$.

\section{Repeated Repair or Replacement}

Ten patients $(17 \%)$ required repeated repair or replacement at a median of 21 months (mean, 22 months; range, 2-62 months) after the AV repair (Figure 2, B). The mechanisms of recurrent $\mathrm{AV}$ insufficiency were as follows: recurrent annular dilatation $(\mathrm{n}=3)$, dysplasia and annular dilatation $(n=4)$, cleft $(n=2)$, and double orifice and annular dilatation $(\mathrm{n}=1)$. Four patients with severely dysplastic leaflets required mechanical valve replacement. Among these 10 patients, prerepair ventricular function was normal in 8 patients, mildly reduced in 1 patient, and moderately reduced in 1 patient. Among the 6 patients who underwent a second $\mathrm{AV}$ repair, residual $\mathrm{AV}$ insufficiency grade was none or trivial in 1 patient, mild in 4 patients, and mild to moderate in 1 patient. Four patients had mild AV stenosis after the operation. The overall survival was $70 \%$ at both 1 and 3 years (causes of deaths listed in Table E4). There was no difference in survival between the repeated repair group and the replacement group $(P=.6)$. One patient who underwent repeated repair had a successful valve replacement 23 months later.

\section{Independent Risks for Repeated Repair or Replacement}

Among the early survivors (survival without transplant more than 6 months after AV repair, $\mathrm{n}=49$ ), younger age at repair $(P=.05)$, smaller BSA $(P=.03)$, increased indexed $\mathrm{AV}$ annular $(P=.002)$ and ventricular $(P=.01)$ dimensions, leaflet dysplasia $(P=.03)$, and residual $\mathrm{AV}$ insufficiency $(P=.05)$ were the significant risk factors for reintervention. Initial diagnosis, ventricular or AV morphology, mechanism of insufficiency, and timing of surgery were not found to be predictors for reintervention (difference not significant for all).

\section{Follow-up}

The median follow-up period was 59 months (mean 52 months, range 1-128 months). The statuses among the 41 long-term survivors are shown in Figure 2, B. The latest echocardiographs of the 34 survivors who as of most recent follow-up still had a single-ventricle physiology with a native $\mathrm{AV}$ showed that $\mathrm{AV}$ insufficiency was none or trivial in 6 patients $(17 \%)$, mild in 23 patients $(67 \%)$, and moderate in 5 patients $(14 \%)$. There was mild AV stenosis in 3 patients $(8 \%)$. Ventricular function was normal in 29 patients $(85 \%)$, mildly reduced in 4 patients $(11 \%)$, and moderately reduced in 1 patient $(8 \%)$. Among 22 patients who had some postrepair ventricular dysfunction, ventricular function was improved in 7 patients $(32 \%)$, unchanged in 10 patients $(45 \%)$, and further reduced in 5 patients $(23 \%)$ at 1 month after AV repair. There was no statistical difference in ventricular function grade over 5 years $(P=.12)$.

\section{Case-Control Outcomes}

Seven patients $(12 \%)$ were excluded from the case-control study because of lack of a matched patient. The remaining 50 patients had complete matching in diagnosis, age, body weight, BSA, and types and timing of palliation according to the criteria. Of the 50 patients, $44(84 \%)$ completely met match criteria. Six had more than 1 grade difference in ventricular function. The comparison of preoperative and postoperative variables between the groups is shown in Table 2 . 
TABLE 2. Patient characteristics in the case-control study

\begin{tabular}{|c|c|c|c|}
\hline Variables & Valve repair & Control & $P$ value \\
\hline Age (mo, median and range) & $6(0-127)$ & $6(0-169)$ & .56 \\
\hline Body weight (kg, median and range) & $5.98(2.1-32)$ & $6(2-32)$ & .39 \\
\hline Male/female ratio & $28: 21$ & $35: 14$ & \\
\hline \multicolumn{4}{|l|}{ Diagnosis (no.) } \\
\hline Hypoplastic left heart syndrome & $32(59 \%)$ & $28(57 \%)$ & \\
\hline Double-outlet right ventricle & $8(15 \%)$ & $7(14 \%)$ & \\
\hline Atrioventricular septal defect & $10(19 \%)$ & $5(10 \%)$ & \\
\hline Tricuspid atresia and double-inlet left ventricle & $4(7 \%)$ & $3(6 \%)$ & \\
\hline Isomerism & $5(9 \%)$ & $2(4 \%)$ & \\
\hline Indeterminate & $0(0 \%)$ & $4(8 \%)$ & \\
\hline \multicolumn{4}{|l|}{ Ventricular morphology (no.) } \\
\hline Right ventricle & $48(84 \%)$ & $43(88 \%)$ & \\
\hline Left ventricle & $7(12 \%)$ & $6(12 \%)$ & \\
\hline Indeterminate & $2(4 \%)$ & $0(0 \%)$ & \\
\hline Preoperative $\mathrm{AV}$ regurgitation grade (mean $\pm \mathrm{SD}$ ) & $2.1 \pm 0.57$ & $0.77 \pm 0.56$ & .0001 \\
\hline Preoperative ventricular function grade (mean \pm SD) & $0.45 \pm 0.69$ & $0.22 \pm 0.55$ & .07 \\
\hline In-hospital death (no.) & $8(14 \%)$ & $2(3.5 \%)$ & .5 \\
\hline Cardiopulmonary bypass (min, median and range) & $118(50-405)$ & $94(8-272)$ & .005 \\
\hline Aortic crossclamp (min, median and range) & $60(15-178)$ & $45.4(14-155)$ & .74 \\
\hline Circulatory arrest & $15(25 \%)$ & $10(20.4 \%)$ & .05 \\
\hline Postoperative AV regurgitation grade & $0.84 \pm 0.62$ & $0.61 \pm 0.57$ & .12 \\
\hline Postoperative ventricular function grade & $0.70 \pm 0.95$ & $0.28 \pm 0.64$ & .01 \\
\hline
\end{tabular}

$A V$, Atrioventricular valve.

Freedom from death or transplant was significantly lower in the valve repair group than in the matched control group (78.9\% vs $92.7 \%$ at 1 year, $68.7 \%$ vs $90.6 \%$ at 3 years, $P=.015$; Figure 3, A). Freedom from all events (death, transplant, repeated repair or replacement) was also significantly lower in the valve repair group than in the matched control group ( $78.8 \%$ vs $92.8 \%$ at 1 year, $58.4 \%$ vs $92.7 \%$ at 3 years, $P=.01$ ). The subgroup analysis, which was performed on the 4 groups determined by postrepair AV insufficiency and ventricular function, showed that group 1 (successful repair with normal ventricular function) had equivalent freedoms both from death or transplant and from all events to the control group (death or transplant $82.7 \%$ vs $89.6 \%$ at 1 year, $76.1 \%$ vs $79.6 \%$ at 3 years, $P=.36$, all events $82.7 \%$ vs $92.4 \%$ at 1 year, $66.9 \%$ vs $81.4 \%$ at 3 years, $P=.19$; Figure $3, B$ ). In group 2 (poor repair with normal ventricular function), both freedom from death or transplant and freedom from all events were significantly lower in the valve repair group than in the control group $(P=.03)$. The numbers of patients in groups 3 and 4 were not sufficient for statistical analysis.

\section{DISCUSSION}

Valve repair resulted in measurably improved valve function regardless of the anatomic lesion. Most of the AV insufficiency ( $85 \%$ ) was related to anatomic abnormalities of the leaflets or subvalvular apparatus. Among the remaining patients who had pure annular dilation (15\%), there was a strong correlation with postrepair ventricular dysfunction and attendant poor survival. Overall survival was significantly worse than that of the case-matched control group, despite early attempts to eliminate insufficiency. Subgroup analysis showed that patients with successful repair and preserved ventricular function $(74 \%$ of the entire cohort) had equivalent early and midterm survivals.

\section{Factors Associated With Death or Transplant}

Our analysis showed preoperative severe annular dilatation (which is highly correlated with ventricular dilatation, $r=.75, P=.0001$ ), CPB time, residual AV insufficiency, and postrepair ventricular function to be predictors for death or transplant. Preoperative morphologic and functional characteristics were unrelated to survival.

Preoperative ventricular dilatation was found to be among the predictors both for failed repair and for all events (death, transplant, reintervention). Preoperative ventricular dilatation grade was also correlated with the postrepair ventricular function $(r=.3, P=.04)$. This phenomenon is common among adult patients undergoing mitral valve repair, in whom the preoperative ventricular dimension predicts postoperative ventricular dysfunction. ${ }^{13}$

The data suggest that in those with AV insufficiency, successful valve intervention for signs of progressive ventricular dilatation could prevent irreversible changes in ventricular geometry and subsequent function impairment. Practically speaking, the use of relatively simple repair 


\section{Freedom from death/transplant Case match control study Entire cohort}

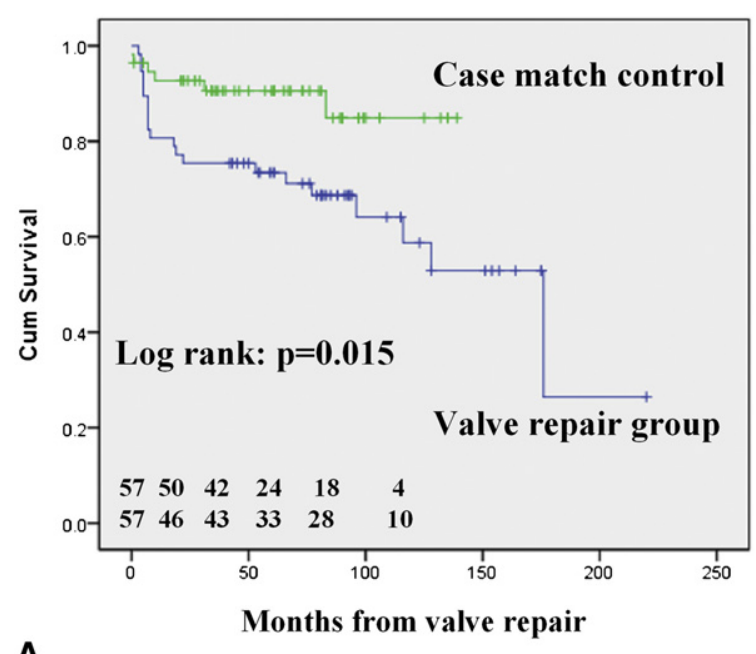

Freedom from death/transplant

Case match control study

Group 1: Successful repair/normal function

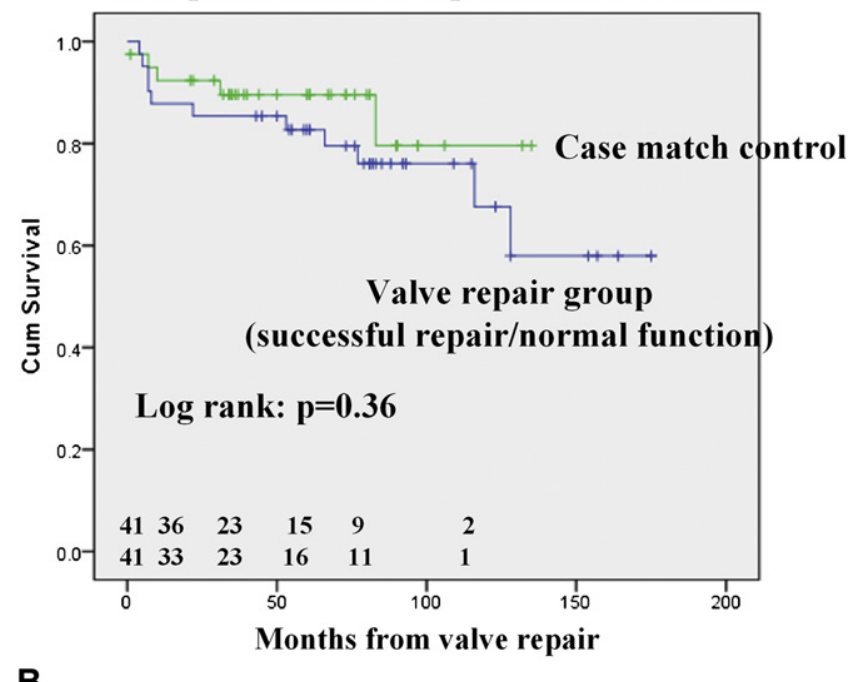

FIGURE 3. Kaplan-Meier survival analysis comparing cumulative (Cum) survivals between the valve repair group and a case-matched control group. A, Entire cohort $(n=56)$. B, The subgroup of the valve repair group with successful repair with normal ventricular function (group 1 , $n=41)$ and its matched control group.

techniques that allow shorter crossclamp and CPB times might be beneficial.

\section{Relationship Between Ventricular Function and AV Insufficiency}

This study showed a strong relationship between postrepair ventricular function and death or transplant. Twelve of the 15 patients who had moderately or severely reduced ventricular function on postrepair TEE $(75 \%)$ eventually reached the end point of mortality or transplant. Chronic volume-related ventricular dysfunction may or may not be reversible by $\mathrm{AV}$ repair. ${ }^{14}$ Progressive postrepair ventricular dysfunction may cause redilatation of the $\mathrm{AV}$ annulus. ${ }^{8}$ Ohye and associates ${ }^{8,11}$ showed that the patients with early and late success of tricuspid valve repair tended to have preserved function and simple repair.

\section{Factors Associated With Repeated Repair or Replacement}

The multivariate logistic regression identified small patients (by age and BSA), dilated annulus and ventricle, leaflet dysplasia, and residual AV insufficiency as predictors for repeated repair or replacement among the early survivors. Furthermore, all 4 patients who had dysplasia as the primary mechanism of reintervention underwent $\mathrm{AV}$ replacement at the second repair. The results indicate that significant residual AV insufficiency associated with dysplastic valve may necessitate subsequent valve replacement. Other ventricular and $\mathrm{AV}$ morphologies were not associated with reintervention.

\section{Durability of Repeated Repair or Replacement for Current AV Insufficiency}

Mahle and colleages ${ }^{15}$ reported the cases of 17 patients who underwent AV replacement. Ten of these 17 patients $(59 \%)$ had previously undergone AV repair. Ohye and associates $^{8,11}$ reported their experiences with repeated repair for residual or recurrent tricuspid valve insufficiency in patients with hypoplastic left heart syndrome. Most of the patients who had failure of the repair had ventricular dysfunction. Subsequent survival of the subgroup was $25 \%$. Our series showed a survival of $70 \%$ at both 1 year and 3 years; however, most had preserved ventricular function.

\section{Timing of AV Repair: Rationale for Early Intervention}

Approximately two thirds of the patients underwent AV repair along with the BCPS. The decision to intervene at stage II for mild to moderate or greater insufficiency has the potential to prevent volume overload related to the regurgitation. The risk of ventricular geometric changes and functional deterioration may be minimized. Mahle and coworkers ${ }^{16}$ suggested a different approach in which they left most patients with moderate or severe AV insufficiency untreated at stage II palliation. Repair was later performed if necessary. They showed equivalent short-term survival between patients with and without significant $\mathrm{AV}$ insufficiency.

For those patients who have significant insufficiency after the BCPS, our strategy is to perform the repair as a standalone procedure if the patient has symptoms. We have 
occasionally combined repair with the Fontan operation, but only if this can be performed simply and efficiently. Fontan physiology results in significant reduction in total cardiac output. ${ }^{2,17}$ Our thinking is that a combination of potential for reduced ventricular function, a lengthy procedure for $\mathrm{AV}$ repair, and the subsequent significant physiologic changes of the Fontan circulation may not be well tolerated.

\section{Effect of Volume Unloading Surgery on AV Insufficiency}

Functional AV insufficiency resulting from chronic volume overload can theoretically be improved by eliminating left-to-right shunt physiology and converting to an in-series circulation of a BCPS at stage II palliation. ${ }^{16,18}$ There is some evidence that a BCPS results in a decrease in the ventricular volume ${ }^{19}$ or the annular dimension ${ }^{20}$; however, previous clinical series showed no or minimal improvement in moderate to severe $\mathrm{AV}$ insufficiency after BCPS. ${ }^{3,16}$ Mahle and coworkers ${ }^{16}$ showed that only 6 of 36 patients who had moderate or severe AV insufficiency at the time of BCPS (22\%) had improved AV insufficiency grade without AV repair. Ten patients (37\%) subsequently required $\mathrm{AV}$ repair. Their findings of need for later repair and minimal improvement by unloading are congruent with our data showing a high incidence of anatomic abnormalities associated with regurgitation. With respect to volume unloading in stage II, recent magnetic resonance imaging data measuring the net left-to-right shunt after a BCPS demonstrate a pulmonary/systemic flow ratio close to $1: 1$ as a result of the development of chest wall collaterals. ${ }^{21}$ The volume unloading of a BCPS therefore appears to be temporary.

\section{Study Limitation}

An ideal matching group would consist of patients with a case match of ventricular function grade and AV insufficiency. Because our policy is to intervene for mild to moderate or greater insufficiency, it was not possible to match for equivalent insufficiency. Therefore this study does not have direct data on the natural history of the patients with mild to moderate or moderate AV insufficiency without AV repair, and positive or negative effects of the early AV repair strategy for this particular subgroup can only be inferred from a multivariate analysis. Magnetic resonance imaging data would allow a more accurate assessment of indices of systolic function and ventricular dilatation.

\section{CONCLUSIONS}

Most $(85 \%)$ of the AV insufficiency in patients with a functionally univentricular heart was related to anatomic abnormalities. Repair measurably improved the grade of insufficiency. Successful repair with preserved postrepair ventricular function was related to a survival equivalent to that of a matched control group. Valve repair for functional $\mathrm{AV}$ insufficiency, which was frequently related to ventricular dysfunction, was not beneficial. Preoperative ventricular dilatation, pure annular dilatation, and postrepair ventricular and valve dysfunction were independent predictors of poor survival or composite outcomes, which suggests that strategies designed to prevent progressive ventricular dilatation and remodeling and to preserve ventricular and valve function would affect clinical outcomes.

\section{References}

1. Choussat A, Fontan F, Besse O. Selection criteria for Fontan's procedure. In: Anderson RH, Shinebourne EA, eds. Pediatric cardiology. Edinburgh: Churchill Livingstone; 1978. p. 559-66.

2. Imai Y, Takanashi Y, Hoshino S, Terada M, Aoki M, Ohta J. Modified Fontan procedure in ninety-nine cases of atrioventricular valve regurgitation. J Thorac Cardiovasc Surg. 1997;113:262-9.

3. Reyes A 2nd, Bove EL, Mosca RS, Kulik TJ, Ludomirsky A. Tricuspid valve repair in children with hypoplastic left heart syndrome during staged surgical reconstruction. Circulation. 1997;96(9 Suppl):II341-5.

4. Hirsch JC, Goldberg C, Bove EL, Salehian S, Lee T, Ohye RG, et al. Fontan operation in the current era: a 15-year single institution experience. Ann Surg. 2008; 248:402-10.

5. Anderson PA, Sleeper LA, Mahony L, Colan SD, Atz AM, Breitbart RE, et al Contemporary outcomes after the Fontan procedure: a Pediatric Heart Network multicenter study. J Am Coll Cardiol. 2008;52:85-98

6. d'Udekem Y, Iyengar AJ, Cochrane AD, Grigg LE, Ramsay JM, Wheaton GR, et al. The Fontan procedure: contemporary techniques have improved longterm outcomes. Circulation. 2007;116(11 Suppl):I157-64.

7. Knott-Craig CJ, Danielson GK, Schaff HV, Puga FJ, Weaver AL, Driscoll DD The modified Fontan operation. An analysis of risk factors for early postoperative death or takedown in 702 consecutive patients from one institution. $J$ Thorac Cardiovasc Surg. 1995;109:1237-43.

8. Ohye RG, Gomez CA, Goldberg CS, Graves HL, Devaney EJ, Bove EL. Tricuspid valve repair in hypoplastic left heart syndrome. J Thorac Cardiovasc Surg. 2004; 127:465-72.

9. Takahashi K, Inage A, Rebeyka IM, Ross DB, Thompson RB, Mackie AS, et al. Real-time 3-dimensional echocardiography provides new insight into mechanisms of tricuspid valve regurgitation in patients with hypoplastic left heart syndrome. Circulation. 2009;120:1091-8.

10. Stamm C, Anderson RH, Ho SY. The morphologically tricuspid valve in hypoplastic left heart syndrome. Eur J Cardiothorac Surg. 1997;12:587-92.

11. Bove EL, Ohye RG, Devaney EJ, Hirsch J. Tricuspid valve repair for hypoplastic left heart syndrome and the failing right ventricle. Semin Thorac Cardiovasc Surg Pediatr Card Surg Annu. 2007;101-4.

12. Ando M, Takahashi Y. Edge-to-edge repair of common atrioventricular or tricuspid valve in patients with functionally single ventricle. Ann Thorac Surg. 2007; 84:1571-7.

13. Matsumura T, Ohtaki E, Tanaka K, Misu K, Tobaru T, Asano R, et al. Echocardiographic prediction of left ventricular dysfunction after mitral valve repair for mitral regurgitation as an indicator to decide the optimal timing of repair. J Am Coll Cardiol. 2003;42:458-63.

14. Pinsky WW, Lewis RM, Hartley CJ, Entman ML. Permanent changes of ventricular contractility and compliance in chronic volume overload. Am J Physiol. 1979;237:H575-83.

15. Mahle WT, Gaynor JW, Spray TL. Atrioventricular valve replacement in patients with a single ventricle. Ann Thorac Surg. 2001;72:182-6.

16. Mahle WT, Cohen MS, Spray TL, Rychik J. Atrioventricular valve regurgitation in patients with single ventricle: impact of the bidirectional cavopulmonary anastomosis. Ann Thorac Surg. 2001;72:831-5.

17. Gewillig M, Brown SC, Eyskens B, Heying R, Ganame J, Budts W, et al. The Fontan circulation: who controls cardiac output? Interact Cardiovasc Thorac Surg. 2010;10:428-33.

18. Freedom RM, Nykanen D, Benson LN. The physiology of the bidirectional cavopulmonary connection. Ann Thorac Surg. 1998;66:664-7. 
19. Forbes TJ, Gajarski R, Johnson GL, Reul GJ, Ott DA, Drescher K, et al. Influence of age on the effect of bidirectional cavopulmonary anastomosis on left ventricular volume, mass and ejection fraction. J Am Coll Cardiol. 1996;28:1301-7.

20. Michelfelder EC, Kimball TR, Beekman RH. Does the superior cavopulmonary anastomosis impact the rate of tricuspid annular dilatation in hypoplastic left heart syndrome? J Am Coll Cardiol. 2001;37:471A.

21. Grosse-Wortmann L, Al-Otay A, Yoo SJ. Aortopulmonary collaterals after bidirectional cavopulmonary connection or Fontan completion: quantification with MRI. Circ Cardiovasc Imaging. 2009;2:219-25.

\section{APPENDIX. Variables for Risk Factor Analysis}

1. Age (months)

2. Sex

3. Body weight at surgery (kilograms)

4. Body surface area (square meters)

5. Diagnosis

6. Isomerism

7. Hypoplastic left heart syndrome

8. Previous Norwood procedure

9. Timing of surgery

10. Ventricular morphology (left, right, indeterminate)

11. Atrioventricular valve morphology

12. Prerepair atrioventricular valve regurgitation grade

13. Atrioventricular valve annulus size (millimeters)

14. Indexed atrioventricular valve annulus size (millimeters per square meter)

15. Affected leaflet: anterior, posterior, septal, mural or lateral, other

16. Regurgitation mechanism: annular dilatation (yes/no)

17. Regurgitation mechanism: cleft (yes/no)

18. Regurgitation mechanism: prolapse (yes/no)

19. Regurgitation mechanism: chordal elongation (yes/no)

20. Regurgitation mechanism: chordal deficiency (yes/no)

21. Regurgitation mechanism: leaflet restriction (yes/no)

22. Regurgitation mechanism: leaflet thickening (yes/no)

23. Regurgitation mechanism: dysplasia (yes/no)

24. Regurgitation mechanism: papillary muscle abnormalities (yes/no)

25. Regurgitation mechanism: endocardial fibroelastosis (yes/no)

26. Pre repair ventricular function (grade $0-3$ )

27. Pre repair ventricular dilatation (grade $0-3$ )

28. Primary mechanism of atrioventricular valve regurgitation

29. Secondary mechanism of atrioventricular valve regurgitation

30. Ventricular dimension (millimeters)

31. Indexed ventricular dimension (millimeters per square meter)

32. Surgical technique: annuloplasty

33. Surgical technique: commissuroplasty

34. Surgical technique: valvuloplasty

35. Surgical technique: chordal repair

36. Surgical technique: cleft closure
37. Surgical technique: edge-to-edge repair

38. Papillary muscle repair

39. Aortic crossclamp time (minutes)

40. Cardiopulmonary bypass time (minutes)

41. Deep hypothermic circulatory arrest (yes/no)

42. Deep hypothermic circulatory arrest time (minutes)

43. Intraoperative transesophageal echocardiogram: residual atrioventricular valve regurgitation (grade $0-3$ )

44. Intraoperative transesophageal echocardiogram: residual atrioventricular valve stenosis (grade $0-3$ )

45. Intraoperative transesophageal echocardiogram: ventricular function (grade $0-3$ )

\section{Discussion}

Dr Jennifer C. Hirsch (Ann Arbor, Mich). Congratulations on a nicely presented article. I am pleased to have the opportunity to discuss this excellent presentation by Dr Honjo and colleagues at The Hospital for Sick Children in Toronto.

As mentioned, the progression of $\mathrm{AV}$ regurgitation in a singleventricle population poses a significant challenge regarding surgical options, timing of intervention, and long-term prognosis. With limited donor availability, every attempt to remediate ventricular and atrioventricular function in the native heart is the ideal objective.

Dr Honjo, you have presented excellent outcomes for patients in whom successful repair has been accomplished. The incorporation of the case-control study provides a valuable attempt to benchmark the outcomes against those of the overall single-ventricle population. Your presentation, as well as the manuscript that you submitted earlier, is thought provoking regarding our own management strategies.

First, it appears that your center has been fairly aggressive at intervening for even mild to moderate AV regurgitation at an early age. We have found significant improvement in the degree of $\mathrm{AV}$ regurgitation with the volume unloading effects of the secondstage cavopulmonary connection, and therefore we have not been intervening for moderate or less $\mathrm{AV}$ regurgitation at that stage. Looking at the relatively small percentage of patients within your study who did have AV repair, how is the decision made to intervene for some of the patients with only mild to moderate regurgitation and not others? And do you know the natural history of those patients who do not have intervention, perhaps through your case-control study?

Dr Honjo. That's a very good point. Our strategy is to take a look at the $\mathrm{AV}$ at stage II palliation if patient has a mild to moderate regurgitation, regardless of the mechanism of regurgitation. Our study shows that the vast majority of patients actually have anatomic abnormality, rather than just functional regurgitation. So it's worth to taking a look at the AV. If there is anatomic issue detected, we can adequately fix it.

We didn't have enough patients with mild to moderate or moderate regurgitation who didn't undergo an AV repair to conduct a good matching study to compare or look at the natural history of unrepaired patient outcome. There is a study from Philadelphia showing that patients with mild to moderate regurgitation, or even moderate regurgitation, who did not have repair at the stage II 
operation did reasonably well after the stage II palliation. The study also suggested, however, that some of these patients required the valve repair at the Fontan operation. We believe that the preservation of ventricle geometry and function is crucial, so our consistent strategy is to intervene in cases of borderline mild to moderate regurgitation at the stage II palliation.

Dr Hirsch. Second, given the high prevalence of single right ventricle and primary tricuspid valve morphologies, do you believe that the development of significant $\mathrm{AV}$ regurgitation is a result of the morphologically right ventricle being incapable of functioning as well under systemic pressures? If so, should these patients be managed differently from a medical standpoint, such as more aggressive afterload reduction to mitigate this risk and stabilize the annulus?

Dr Honjo. I think that's a good point. In our patient group, more than $80 \%$ of the patients have morphologically right ventricle with either anatomic tricuspid valve or common AV. If they start to have AV regurgitation or progressive ventricular dilatation, they need aggressive medical therapy to reduce afterload. We believe that to intervene for those patients at stage II is part of an overall strategy that will minimize irreversible changes in ventricular function and geometry.

Dr Hirsch. Given the variable approaches to annuloplasty and partial annuloplasty in this patient population that have been reported, do you use a standard technique for your annuloplasty? If so, what is that technique?

Dr Honjo. We used very standard techniques. We only use the ring annuloplasty or circumferential annuloplasty when the annulus is at or near adult size. More than $95 \%$ of the patients actually have localized annuloplasty where the leak originates. We also combine either anatomic functional commissuroplasty at the location of the regurgitation. Some patients underwent edge-to-edge type repairs, especially the patient with common AV.

Dr Hirsch. Do you think that with the coming availability of the bioabsorbable ring that perhaps you'll use that more frequently in this patient population to further stabilize the annulus?

Dr Honjo. You mean the artificial ring?

Dr Hirsch. Bioabsorbable.

Dr Honjo. I'm not sure.

Dr Hirsch. It's not yet commercially available. I'm thinking in the future of new technologies that may benefit these patients.

Dr Honjo. Right. That might be a good idea.

Dr Hirsch. Finally, given the increased risk of failure in your patients with poor ventricular function and significant annular dilatation, do you believe that early listing for transplant may be appropriate for this patient population?
Dr Honjo. That's a good point. I think that we have to consider transplant early when we have a patient who has reduced ventricular function after AV repair. Those are the patients who are at really high risk of death or transplant anyway. The issue is that we cannot predict who is going to have postrepair severe ventricular dysfunction according to the preoperative values in our study. That's one thing we have to sort out in a future study.

Dr Hirsch. Again, congratulations on an outstanding presentation and exemplary results in a very difficult patient population.

Dr Honjo. Thank you very much.

Dr Peter B. Manning (Cincinnati, Ohio). You said that most of your valves were anatomically abnormal and that most of your patients had hypoplastic left heart syndrome, and I don't think we usually think of patients with hypoplastic left heart syndrome as having anatomically abnormal tricuspid valves. Are you basing the characterization of abnormal on the fact that you had a high percentage of annular dilatation and prolapse according to echocardiography? Annular dilatation with reference to what normal value? I'm guessing there probably aren't normal values for single-ventricle annular dimensions. With your case-control study, you might have a comparison group to justify that.

I also worry that prolapse may also be a function more of ventricular dysfunction than of actual anatomic findings. So were the surgical intraoperative findings consistent with what you're characterizing as echocardiographic anatomic abnormalities? That is my first question.

Dr Honjo. That's a very good point. We actually are performing an ongoing study correlating the surgical and echocardiographic findings in this study. We do, however, have a fair correlation between the evaluation of the echocardiograms that were done in this study and actual surgical findings. The pathologic study also showed that more than a third of the patients with hypoplastic left heart syndrome actually had anatomic abnormality, mainly dysplasia, so it's not surprising that the vast majority of patients in this patient group had anatomic abnormalities.

Dr Manning. I think that the whole problem is a difficult chicken-and-egg situation sometimes. Is the valve regurgitation the problem, or is it the ventricular dysfunction that's the primary problem? Perhaps, particularly with some of these patients, what you're showing is there is a significant red flag. and you can repair the valve all you want but the ventricle is still the problem and that's failing.

I think this is very useful information, and further long-term follow-up looking at trying to get these kids out to 20 and 30 years is really what we're all striving for. 
Pre-repair (2.1+/-0.57)

Post-repair $(0.84+/-0.62)$

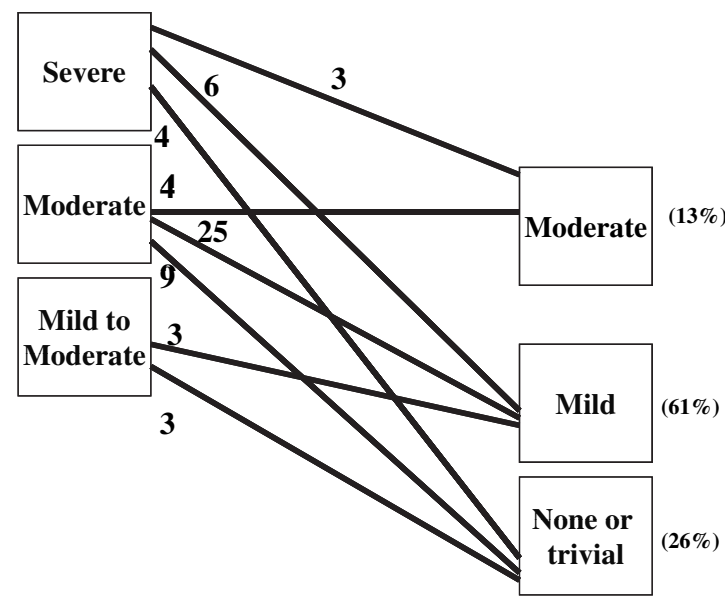

FIGURE E1. Changes in atrioventricular valve regurgitation grade before and after atrioventricular valve repair. The regurgitation grade was significantly reduced by the atrioventricular valve repair. Three of the 13 patients with severe atrioventricular valve insufficiency $(23 \%)$ and 4 of the 38 patients with moderate atrioventricular valve insufficiency $(10 \%)$ had moderate residual atrioventricular valve insufficiency $(12 \%$ of the entire cohort). All the patients with mild to moderate regurgitation showed improvement to mild or trivial regurgitation.
TABLE E2. Grading of atrioventricular valve insufficiency, stenosis, and ventricular function, and subgroups according to postrepair atrioventricular valve and ventricular function

\begin{tabular}{cl}
\hline Grade or group & \multicolumn{1}{c}{ Description } \\
\hline Atrioventricular valve insufficiency \\
0 & None or trivial \\
1 & Mild \\
2 & Moderate \\
3 & Severe \\
Atrioventricular valve stenosis \\
0 & No inflow pressure gradient \\
1 & Mild (mean pressure gradient $<5 \mathrm{~mm} \mathrm{Hg}$ ) \\
2 & Moderate (mean pressure gradient 5-10 mm Hg) \\
3 & Severe (mean pressure gradient $>10 \mathrm{~mm} \mathrm{Hg})$ \\
Ventricular function & \\
0 & Normal \\
1 & Mildly reduced \\
2 & Moderately reduced \\
3 & Severely reduced \\
Subgroup & Successful repair (no more than mild to \\
Group 1 & moderate regurgitation) with normal \\
& ventricular function \\
Group 2 & Poor repair (at least moderate regurgitation) \\
Group 3 & with normal ventricular function \\
Group 4 & Successful repair with poor ventricular \\
\hline & function (at least moderately reduced) \\
& Poor repair with poor ventricular function \\
\hline
\end{tabular}

TABLE E1. Preoperative profile

\begin{tabular}{lc}
\hline No. of patients & 57 \\
Age (mo, median and range) & $6.8(0.3-209)$ \\
Body weight (kg, median and range) & $6.95(2.1-58.8)$ \\
Male/female ratio & $32: 26$ \\
Diagnosis (no.) & \\
$\quad$ Hypoplastic left heart syndrome & $32(59 \%)$ \\
Double-outlet right ventricle & $8(15 \%)$ \\
Atrioventricular septal defect & $10(19 \%)$ \\
Tricuspid atresia and double-inlet left ventricle & $4(7 \%)$ \\
Isomerism & $5(9 \%)$ \\
Ventricular morphology (no.) & \\
Right ventricle & $48(83 \%)$ \\
Left ventricle & $8(13 \%)$ \\
Indeterminate & $2(4 \%)$ \\
Timing of valve repair (no.) & \\
Stage I (Norwood) & $2(3 \%)$ \\
Stage II (bidirectional cavopulmonary shunt) & $33(59 \%)$ \\
Between stage II and III & $10(17 \%)$ \\
Stage III (Fontan) & $10(17 \%)$ \\
After stage III & $2(3 \%)$ \\
\hline
\end{tabular}


TABLE E3. Repair techniques, operative variables, and postrepair echocardiographic findings

\begin{tabular}{lc}
\hline \multicolumn{1}{c}{ Variables } \\
\hline Operative techniques (no.) & \\
Annuloplasty & $46(85 \%)$ \\
Commissuroplasty & $29(54 \%)$ \\
Valvuloplasty & $12(22 \%)$ \\
Chordal repair & $2(4 \%)$ \\
Cleft closure & $18(33 \%)$ \\
Edge-to-edge repair & $17(29 \%)$ \\
Papillary muscle repair & $1(2 \%)$ \\
Operative variables & \\
Cardiopulmonary bypass (min, median and range) & $118(50-405)$ \\
Crossclamp (min, median and range) & $60(15-178)$ \\
Circulatory arrest (no.) & $15(25 \%)$ \\
Circulatory arrest time (min, median and range) & $22(2-67)$ \\
Residual atrioventricular valve insufficiency (no.) & \\
None or trivial & $14(26 \%)$ \\
Mild & $33(61 \%)$ \\
Moderate & $7(13 \%)$ \\
Postrepair atrioventricular valve stenosis (no.) & \\
None or trivial & $45(83 \%)$ \\
Mild & $8(15 \%)$ \\
Moderate & $1(2 \%)$ \\
Postrepair ventricular function & \\
Normal & $36(63 \%)$ \\
Mildly reduced & $10(17 \%)$ \\
Moderately reduced & $7(13 \%)$ \\
Severely reduced & $4(7 \%)$ \\
\hline
\end{tabular}

TABLE E4. Causes of early and late deaths

\begin{tabular}{lc}
\hline \multicolumn{1}{c}{ Cause of death } & No. \\
\hline All-cause in-hospital deaths & 8 \\
Moderate residual atrioventricular valve insufficiency & \\
$\quad$ Sudden arrest & 1 \\
$\quad$ Progressive ventricular failure & 1 \\
Poor myocardial protection & 1 \\
Hypoplastic left heart syndrome after Norwood procedure & \\
$\quad$ Cardiac arrest & 2 \\
Hypoxia, sepsis, supraventricular tachycardia after & 1 \\
$\quad$ second valve repair & \\
Superior vena cava thrombosis & 1 \\
Fontan takedown, low cardiac output & 1 \\
All-cause late deaths & 9 \\
Mechanical valve replacement related & \\
Stuck valve after atrioventricular valve & 1 \\
$\quad$ replacement, ventricular dysfunction & \\
Gastrointestinal bleeding on postoperative & 1 \\
$\quad$ extracorporeal membrane oxygenation after & \\
$\quad$ atrioventricular valve replacement & \\
After heart transplant & \\
Acute rejection & \\
Respiratory arrest & \\
Failed Fontan, cardiac arrest & \\
Sudden death at home & \\
Multiple venous thrombosis & 1 \\
Pneumonia, sepsis & 1 \\
Pulmonary hypertension, pulmonary vein stenosis, infection & 1 \\
\hline
\end{tabular}

